\title{
An algorithm for finding common solutions of various problems in nonlinear operator theory
}

\author{
Eric U Ofoedu' ${ }^{1}$, Jonathan N Odumegwu' ${ }^{1}$ Habtu Zegeye ${ }^{2}$ and Naseer Shahzad ${ }^{3 *}$
}

\section{"Correspondence: \\ nshahzad@kau.edu.sa \\ ${ }^{3}$ Department of Mathematics, King \\ Abdulaziz University, P.O. Box 80203 \\ Jeddah, 21589, Saudi Arabia \\ Full list of author information is \\ available at the end of the article}

\begin{abstract}
In this paper, it is our aim to prove strong convergence of a new iterative algorithm to a common element of the set of solutions of a finite family of classical equilibrium problems; a common set of zeros of a finite family of inverse strongly monotone operators; the set of common fixed points of a finite family of quasi-nonexpansive mappings; and the set of common fixed points of a finite family of continuous pseudocontractive mappings in Hilbert spaces on assumption that the intersection of the aforementioned sets is not empty. Moreover, the common element is shown to be the metric projection of the initial guess on the intersection of these sets.
\end{abstract}

MSC: 47H06; 47H09; 47J05; 47J25

Keywords: classical equilibrium problem; generalized mixed equilibrium problem; $\eta$-inverse strongly monotone mapping; maximal monotone operator; nonexpansive mappings; real Hilbert space; pseudocontractive mappings; variational inequality problem

\section{Introduction}

Let $H$ be a real Hilbert space. A mapping $T$ with domain $D(T)$ and range $R(T)$ in $H$ is called an L-Lipschitzian mapping (or simply a Lipschitz mapping) if and only if there exists $L \geq 0$ such that for all $x, y \in D(T)$,

$$
\|T x-T y\| \leq L\|x-y\| .
$$

If $L \in[0,1)$, then $T$ is called strict contraction or simply a contraction; and if $L=1$, then $T$ is called nonexpansive. A point $x \in D(T)$ is called a fixed point of an operator $T$ if and only if $T x=x$. The set of fixed points of an operator $T$ is denoted by $\operatorname{Fix}(T)$, that is, $\operatorname{Fix}(T):=$ $\{x \in D(T): T x=x\}$.

A mapping $T$ with domain $D(T)$ and range $R(T)$ in $H$ is called a quasi-nonexpansive mapping if and only if $\operatorname{Fix}(T) \neq \emptyset$ and for any $x \in D(T)$, for any $u \in \operatorname{Fix}(T)$,

$$
\|T x-u\| \leq\|x-u\|
$$

Every nonexpansive mapping with a nonempty fixed point set is quasi-nonexpansive. The following examples show that the converse is not true.

(c)2014 Ofoedu et al.; licensee Springer. This is an Open Access article distributed under the terms of the Creative Commons Attribution License (http://creativecommons.org/licenses/by/2.0), which permits unrestricted use, distribution, and reproduction in any medium, provided the original work is properly cited. 
Example 1.1 (see [1]) Let $E=[-\pi, \pi]$ be a subspace of the set of real numbers $\mathbb{R}$, endowed with the usual topology. Define $T: E \rightarrow E$ by $T x=x \cos x$ for all $x \in E$. Clearly, $F(T)=\{0\}$. Observe that

$$
|T x-0|=|x| \times|\cos x| \leq|x|=|x-0| .
$$

Thus, $T$ is quasi-nonexpansive. The mapping $T$ is, however, not a nonexpansive mapping since for $x=\frac{\pi}{2}$ and $y=\pi$,

$$
|T x-T y|=\left|\frac{\pi}{2} \cos \left(\frac{\pi}{2}\right)-\pi \cos \pi\right|=\pi .
$$

But

$$
|x-y|=\left|\frac{\pi}{2}-\pi\right|=\frac{\pi}{2} .
$$

Example 1.2 (see $[1,2])$ Let $E=\mathbb{R}$ be endowed with usual topology. Define $T: \mathbb{R} \rightarrow \mathbb{R}$ by

$$
T x= \begin{cases}\frac{x}{2} \cos \left(\frac{1}{x}\right), & x \neq 0, \\ 0, & x=0 .\end{cases}
$$

It is easy to see that $F(T)=\{0\}$ since for $x \neq 0, T x=x$ implies that $\frac{x}{2} \cos \frac{1}{x}=x$. Thus, for any $x \neq 0, \cos \frac{1}{x}=2$, which is not possible. So, $F(T)=\{0\}$. Next, observe that for any $x \in \mathbb{R}$,

$$
|T x-0|=\left|\frac{x}{2}\right| \times\left|\cos \left(\frac{1}{x}\right)\right| \leq \frac{|x|}{2}<|x|=|x-0| .
$$

So, the mapping $T$ is quasi-nonexpansive. Finally, we show that $T$ is not nonexpansive. To see this, let $x=\frac{2}{3 \pi}$ and $y=\frac{1}{\pi}$, then

$$
|T x-T y|=\left|\frac{1}{3 \pi} \cos \left(\frac{3 \pi}{2}\right)-\frac{1}{2 \pi} \cos \pi\right|=\frac{1}{2 \pi} .
$$

But,

$$
|x-y|=\left|\frac{2}{3 \pi}-\frac{1}{\pi}\right|=\frac{1}{3 \pi} .
$$

So,

$$
|T x-T y|=\frac{1}{2 \pi}>\frac{1}{3 \pi}=|x-y| .
$$

The concept of quasi-nonexpansive mappings was essentially introduced by Diaz and Metcalf [3]. Although Examples 1.1 and 1.2 guarantee the existence of a quasinonexpansive mapping which is not nonexpansive, we must note that a linear quasinonexpansive mapping defined on a subspace of a given vector space is nonexpansive on that subspace. 
Another important generalization of the class of nonexpansive mappings is the class of pseudocontractive mappings. These mappings are intimately connected with the important class of nonlinear accretive operators. This connection will be made precise in what follows.

A mapping $T$ with domain $D(T)$ and range $R(T)$ in $H$ is called pseudocontractive if and only if for all $x, y \in D(T)$, the following inequality holds:

$$
\|x-y\| \leq\|(1+r)(x-y)-r(T x-T y)\|
$$

for all $r>0$. As a consequence of a result of Kato [4], the pseudocontractive mappings can also be defined in terms of the normalized duality mappings as follows: the mapping $T$ is called pseudocontractive if and only if for all $x, y \in D(T)$, we have that

$$
\langle T x-T y, x-y\rangle \leq\|x-y\|^{2} .
$$

It now follows trivially from (1.3) that every nonexpansive mapping is pseudocontractive. We note immediately that the class of pseudocontractive mappings is larger than that of nonexpansive mappings. For examples of pseudocontractive mappings which are not nonexpansive, the reader may see [5].

To see the connection between the pseudocontractive mappings and the monotone mappings, we introduce the following definition: a mapping $A$ with domain $D(A)$ and range $R(A)$ in $E$ is called monotone if and only if for all $x, y \in D(A)$, the following inequality is satisfied:

$$
\langle A x-A y, x-y\rangle \geq 0 .
$$

The operator $A$ is called $\eta$-inverse strongly monotone if and only if there exists $\eta \in(0,1)$ such that for all $x, y \in D(A)$, we have that

$$
\langle A x-A y, x-y\rangle \geq \eta\|A x-A y\|^{2} .
$$

It is easy to see from inequalities (1.3) and (1.4) that an operator $A$ is monotone if and only if the mapping $T:=(I-A)$ is pseudocontractive. Consequently, the fixed point theory for pseudocontractive mappings is intimately connected with the zero of monotone mappings. For the importance of monotone mappings and their connections with evolution equations, the reader may consult any of the references $[5,6]$.

Due to the above connection, fixed point theory of pseudocontractive mappings became a flourishing area of intensive research for several authors.

Let $C$ be a closed convex nonempty subset of a real Hilbert space $H$ with inner product $\langle\cdot, \cdot\rangle$ and norm $\|\cdot\|$. Let $f: C \times C \rightarrow \mathbb{R}$ be a bifunction. The classical equilibrium problem $(E P)$ for a bifunction $f$ is to find $u^{*} \in C$ such that

$$
f\left(u^{*}, y\right) \geq 0, \quad \forall y \in C .
$$

The set of solutions for $E P(1.6)$ is denoted by

$$
E P(f)=\{u \in C: f(u, y) \geq 0, \forall y \in C\} .
$$


The classical equilibrium problem $(E P)$ includes as special cases the monotone inclusion problems, saddle point problems, variational inequality problems, minimization problems, optimization problems, vector equilibrium problems, Nash equilibria in noncooperative games. Furthermore, there are several other problems, for example, the complementarity problems and fixed point problems, which can also be written in the form of the classical equilibrium problem. In other words, the classical equilibrium problem is a unifying model for several problems arising from engineering, physics, statistics, computer science, optimization theory, operations research, economics and countless other fields. For the past 20 years or so, many existence results have been published for various equilibrium problems (see, e.g., [7-10]). Approximation methods for such problems thus become a necessity.

Iterative approximation of fixed points and zeros of nonlinear mappings has been studied extensively by many authors to solve nonlinear mapping equations as well as variational inequality problems and their generalizations (see, e.g., [11-19]). Most published results on nonexpansive mappings (for example) focus on the iterative approximation of their fixed points or approximation of common fixed points of a given family of this class of mappings.

Some attempts to modify the Mann iteration method so that strong convergence is guaranteed have recently been made (we should recall that Mann iteration method only guarantees weak convergence (see, for example, Bauschke et al. [20])). Nakajo and Takahashi [16] formulated the following modification of the Mann iteration method for a nonexpansive mapping $T$ defined on a nonempty bounded closed and convex subset $C$ of a Hilbert space $H$ :

$$
\left\{\begin{array}{l}
x_{0} \in C, \\
y_{n}=\alpha_{n} x_{n}+\left(1-\alpha_{n}\right) T x_{n}, \\
C_{n}=\left\{v \in C:\left\|y_{n}-v\right\|^{2} \leq\left\|x_{n}-v\right\|^{2}\right\}, \\
Q_{n}=\left\{v \in C:\left\langle x_{n}-v, x_{0}-x_{n}\right\rangle \geq 0\right\}, \\
x_{n+1}=P_{C_{n} \cap Q_{n}}\left(x_{0}\right), \quad \forall n \in \mathbb{N},
\end{array}\right.
$$

where $P_{C}$ denotes the metric projection from $H$ onto a closed convex subset $C$ of $H$. They proved that if the sequence $\left\{\alpha_{n}\right\}_{n \geq 0}$ is bounded away from 1 , then $\left\{x_{n}\right\}_{n \geq 0}$ defined by (1.7) converges strongly to $P_{F(T)}\left(x_{0}\right)$.

Formulations similar to (1.7) for different classes of nonlinear problems had been presented by Kim and $\mathrm{Xu}$ [21], Nilsrakoo and Saejung [22], Ofoedu et al. [23], Yang and Su [24], Zegeye and Shahzad [25-27].

In this paper, motivated by the results of the authors mentioned above, it is our aim to prove strong convergence of a new iterative algorithm to a common element of the set of solutions of a finite family of classical equilibrium problems; a common set of zeros of a finite family of inverse strongly monotone mappings; a set of common fixed points of a finite family of quasi-nonexpansive mappings; and a set of common fixed points of a finite family of continuous pseudocontractive mappings in Hilbert spaces on assumption that the intersection of the aforementioned sets is not empty. Moreover, the common element is shown to be the metric projection of the initial guess on the intersection of these sets. Our theorems complement the results of the authors mentioned above and those of several other authors. 


\section{Preliminary}

In what follows, we shall make use of the following lemmas.

Lemma 2.1 (see, e.g., Kopecka and Reich [28]) Let $C$ be a nonempty closed and convex subset of a real Hilbert space. Let $x \in H$ and $P_{C}: H \rightarrow C$ be the metric projection of $H$ onto $C$, then for any $y \in C$,

$$
\left\|y-P_{C} x\right\|^{2}+\left\|P_{C} x-x\right\|^{2} \leq\|x-y\|^{2} .
$$

Lemma 2.2 Let $C$ be a closed convex nonempty subset of a real Hilbert space $H$; and let $P_{C}: H \rightarrow C$ be the metric projection of $H$ onto $C$. Let $x \in H$, then $x_{0}=P_{C} x$ if and only if $\left\langle z-x_{0}, x-x_{0}\right\rangle \leq 0$ for all $z \in C$.

Lemma 2.3 Let $H$ be a real Hilbert space, then for any $x, y \in H, \alpha \in[0,1]$,

$$
\|\alpha x+(1-\alpha) y\|^{2}=\alpha\|x\|^{2}+(1-\alpha)\|y\|^{2}-\alpha(1-\alpha)\|x-y\|^{2} .
$$

Lemma 2.4 (see Zegeye [29]) Let $C$ be a nonempty closed convex subset of a real Hilbert space $H$. Let $T: C \rightarrow H$ be a continuous pseudocontractive mapping, then for all $r>0$ and $x \in H$, there exists $z \in C$ such that

$$
\langle y-z, T z\rangle-\frac{1}{r}\langle y-z,(1+r) z-x\rangle \leq 0, \quad \forall y \in C
$$

Lemma 2.5 (see Zegeye [29]) Let $C$ be a nonempty closed convex subset of a real Hilbert space $H$. Let $T: C \rightarrow C$ be a continuous pseudocontractive mapping, then for all $r>0$ and $x \in H$, define a mapping $F_{r}: H \rightarrow C$ by

$$
F_{r} x=\left\{z \in C:\langle y-z, T z\rangle-\frac{1}{r}\langle y-z,(1+r) z-x\rangle \leq 0, \forall y \in C\right\}
$$

then the following hold:

(1) $F_{r}$ is single-valued;

(2) $F_{r}$ is firmly nonexpansive type mapping, i.e., for all $x, y \in H$,

$$
\left\|F_{r} x-F_{r} y\right\|^{2} \leq\left\langle F_{r} x-F_{r} y, x-y\right\rangle
$$

(3) $\operatorname{Fix}\left(F_{r}\right)$ is closed and convex; and $\operatorname{Fix}\left(F_{r}\right)=\operatorname{Fix}(T)$ for all $r>0$.

In the sequel, we shall require that the bifunction $f: C \times C \rightarrow \mathbb{R}$ satisfies the following conditions:

(A1) $f(x, x)=0, \forall x \in C$;

(A2) $f$ is monotone in the sense that $f(x, y)+f(y, x) \leq 0$ for all $x, y \in C$;

(A3) $\limsup _{t \rightarrow 0^{+}} f(t z+(1-t) x, y) \leq f(x, y)$ for all $x, y, z \in C$;

(A4) the function $y \mapsto f(x, y)$ is convex and lower semicontinuous for all $x \in C$.

Lemma 2.6 (see, e.g., $[7,30]$ ) Let $C$ be a closed convex nonempty subset of a real Hilbert space $H$. Let $f: C \times C \rightarrow \mathbb{R}$ be a bifunction satisfying conditions (A1)-(A4), then for all 
$r>0$ and $x \in H$, there exists $u \in C$ such that

$$
f(u, y)+\frac{1}{r}\langle y-u, u-x\rangle \geq 0, \quad \forall y \in C .
$$

Moreover, if for all $x \in H$ we define a mapping $G_{r}: H \rightarrow 2^{C}$ by

$$
G_{r}(x)=\left\{u \in C: f(u, y)+\frac{1}{r}\langle y-u, u-x\rangle \geq 0, \forall y \in C\right\},
$$

then the following hold:

(1) $G_{r}$ is single-valued for all $r>0$;

(2) $G_{r}$ is firmly nonexpansive, that is, for all $x, z \in H$,

$$
\left\|G_{r} x-G_{r} z\right\|^{2} \leq\left\langle G_{r} x-G_{r} z, x-z\right\rangle ;
$$

(3) $\operatorname{Fix}\left(G_{r}\right)=E P(f)$ for all $r>0$;

(4) $E P(f)$ is closed and convex.

Lemma 2.7 (see Ofoedu [31]) Let $C$ be a nonempty closed convex subset of a real Hilbert space H. Let $T: C \rightarrow C$ be a continuous pseudocontractive mapping. For $r>0$, let $F_{r}: H \rightarrow$ $C$ be the mapping in Lemma 2.5, then for any $x \in H$ and for any $p, q>0$,

$$
\left\|F_{p} x-F_{q} x\right\| \leq \frac{|p-q|}{p}\left(\left\|F_{p} x\right\|+\|x\|\right)
$$

Lemma 2.8 (Compare with Lemma 13 of Ofoedu [31]) Let $C$ be a closed convex nonempty subset of a real Hilbert space $H$. Let $f: C \times C \rightarrow \mathbb{R}$ be a bifunction satisfying conditions (A1)-(A4). Let $r>0$ and let $G_{r}$ be the mapping in Lemma 2.6, then for all $p, q>0$ and for all $x \in H$, we have that

$$
\left\|G_{p} x-G_{q} x\right\| \leq \frac{|p-q|}{p}\left(\left\|G_{p} x\right\|+\|x\|\right) .
$$

\section{Main results}

Let $C$ be a nonempty closed convex subset of a real Hilbert space $H$. Let $T_{1}, T_{2}, \ldots, T_{m}$ : $C \rightarrow C$ be $m$ continuous pseudocontractive mappings; let $S_{1}, S_{2}, \ldots, S_{l}: C \rightarrow C$ be $l$ continuous quasi-nonexpansive mappings; let $A_{1}, A_{2}, \ldots, A_{d}: C \rightarrow H$ be $d \gamma_{j}$-inverse strongly monotone mappings with constants $\gamma_{j} \in(0,1), j=1,2, \ldots, d$; let $f_{1}, f_{2}, \ldots, f_{t}: C \times C \rightarrow \mathbb{R}$ be $t$ bifunctions satisfying conditions (A1)-(A4). For all $x \in E, i=1,2, \ldots, m$, let

$$
F_{i, r} x:=\left\{z \in C:\left\langle y-z, T_{i} z\right\rangle-\frac{1}{r}\langle y-z,(1+r) z-x\rangle \leq 0, \forall y \in C\right\}
$$

and for all $x \in E, h=1,2, \ldots, t$, let

$$
G_{h, r}(x)=\left\{u \in C: f_{h}(u, y)+\frac{1}{r}\langle y-u, u-x\rangle \geq 0, \forall y \in C\right\},
$$


then in what follows we shall study the following iteration process:

$$
\left\{\begin{array}{l}
x_{0} \in C_{0}=C \quad \text { chosen arbitrarily, } \\
z_{n}=P_{C}\left(x_{n}-\lambda_{n} A_{n+1} x_{n}\right), \\
y_{n}=\alpha_{n} x_{n}+\left(1-\alpha_{n}\right) S_{n+1} z_{n}, \\
w_{n}=\eta \sum_{i=1}^{m} \beta_{i} F_{i, r_{n}} y_{n}+(1-\eta) \sum_{h=1}^{t} \xi_{h} G_{h, r_{n}} y_{n}, \\
C_{n+1}=\left\{z \in C:\left\|w_{n}-z\right\| \leq\left\|x_{n}-z\right\|\right\}, \\
x_{n+1}=\Pi_{C_{n+1}}\left(x_{0}\right), \quad n \geq 0,
\end{array}\right.
$$

where $A_{n}=A_{n(\bmod d)}, S_{n}=S_{n(\bmod l)} ;\left\{r_{n}\right\} \subset(0, \infty)$ such that $\lim _{n \rightarrow \infty} r_{n}=r_{0}>0 ;\left\{\alpha_{n}\right\}_{n \geq 1}$ a sequence in $(0,1)$ such that $\liminf _{n \rightarrow \infty} \alpha_{n}\left(1-\alpha_{n}\right)>0 ;\left\{\beta_{i}\right\}_{i=1}^{m},\left\{\xi_{h}\right\}_{h=1}^{t} \subset(0,1)$ such that $\sum_{i=1}^{m} \beta_{i}=1=\sum_{h=1}^{t} \xi_{h} ; \eta \in(0,1)$ and $\left\{\lambda_{n}\right\}$ is a sequence in $[a, b]$ for some $a, b \in \mathbb{R}$ such that $0<a<b<2 \gamma, \gamma=\min _{1 \leq j \leq d}\left\{\gamma_{j}\right\}$.

Lemma 3.1 Let $C$ be a nonempty closed convex subset of a real Hilbert space $H$. Let $T_{1}, T_{2}, \ldots, T_{m}: C \rightarrow C$ be $m$ continuous pseudocontractive mappings; let $S_{1}, S_{2}, \ldots, S_{l}$ : $C \rightarrow C$ be l continuous quasi-nonexpansive mappings; let $A_{1}, A_{2}, \ldots, A_{d}: C \rightarrow H$ be $d \gamma_{j}$ inverse strongly monotone mappings with constants $\gamma_{j} \in(0,1), j=1,2, \ldots, d ;$ let $f_{1}, f_{2}, \ldots, f_{t}$ : $C \times C \rightarrow \mathbb{R}$ be $t$ bifunctions satisfying conditions (A1)-(A4). Let $F:=\bigcap_{i=1}^{m} \operatorname{Fix}\left(T_{i}\right) \cap$ $\bigcap_{j=1}^{d} A_{j}^{-1}(0) \cap \bigcap_{k=1}^{l} \operatorname{Fix}\left(S_{k}\right) \cap \bigcap_{h=1}^{t} E P\left(f_{h}\right) \neq \emptyset$. Let $\left\{x_{n}\right\}$ be a sequence defined by (3.1), then the sequence $\left\{x_{n}\right\}$ is well defined for each $n \geq 0$.

Proof We first show that $C_{n}$ is closed and convex for each $n \in \mathbb{N} \cup\{0\}$. From the definitions of $C_{n}$ it is obvious that $C_{n}$ is closed. Moreover, since $\left\|w_{n}-z\right\| \leq\left\|x_{n}-z\right\|$ is equivalent to $2\left\langle z, x_{n}-w_{n}\right\rangle-\left\|x_{n}\right\|^{2}+\left\|w_{n}\right\|^{2} \leq 0$, it follows that $C_{n}$ is convex for each $n \in \mathbb{N} \cup\{0\}$. Next, we prove that $F \subset C_{n}$ for each $n \in \mathbb{N} \cup\{0\}$. From the assumption, we see that $F \subset C_{0}=C$. Suppose that $F \subset C_{k}$ for some $k \geq 1$, then for $p \in F$, we obtain that

$$
\begin{aligned}
\left\|w_{k}-p\right\| & =\left\|\eta \sum_{i=1}^{m} \beta_{i} F_{i, r_{k}} y_{k}+(1-\eta) \sum_{h=1}^{m} \xi_{h} G_{h, r_{k}} y_{k}-p\right\| \\
& \leq\left\|y_{k}-p\right\|=\left\|\alpha_{k} x_{k}+\left(1-\alpha_{k}\right) S_{k+1} z_{k}-p\right\| \\
& \leq \alpha_{k}\left\|x_{k}-p\right\|+\left(1-\alpha_{k}\right)\left\|S_{k+1} z_{k}-p\right\| \\
& \leq \alpha_{k}\left\|x_{k}-p\right\|+\left(1-\alpha_{k}\right)\left\|z_{k}-p\right\| .
\end{aligned}
$$

Furthermore,

$$
\begin{aligned}
\left\|z_{k}-p\right\|^{2} & =\left\|P_{C}\left(x_{k}-\lambda_{k} A_{k+1} x_{k}\right)-p\right\|^{2} \\
& \leq\left\|x_{k}-\lambda_{k} A_{k+1} x_{k}-p\right\|^{2} \\
& =\left\|x_{k}-p-\lambda_{k}\left(A_{k+1} x_{k}-A_{k+1} p\right)\right\|^{2} \\
& =\left\|x_{k}-p\right\|^{2}-2 \lambda_{k}\left\langle x_{k}-p, A_{k+1} x_{k}-A_{k+1} p\right\rangle+\lambda_{k}^{2}\left\|A_{k+1} x_{k}-A_{k+1} p\right\|^{2} \\
& \leq\left\|x_{k}-p\right\|^{2}+\lambda_{k}\left(\lambda_{k}-2 \gamma\right)\left\|A_{k+1} x_{k}-A_{k+1} p\right\|^{2} \\
& \leq\left\|x_{k}-p\right\|^{2} \quad\left(\text { since } \lambda_{k}<2 \gamma\right) .
\end{aligned}
$$


Thus,

$$
\left\|z_{k}-p\right\| \leq\left\|x_{k}-p\right\|
$$

Using (3.3) in (3.2) gives

$$
\left\|w_{k}-p\right\| \leq\left\|x_{k}-p\right\|
$$

So, $p \in C_{k+1}$. This implies, by induction, that $F \subset C_{n}$ so that the sequence generated by (3.1) is well defined for all $n \geq 0$.

Theorem 3.2 Let $C$ be a nonempty closed convex subset of a real Hilbert space $H$. Let $T_{1}, T_{2}, \ldots, T_{m}: C \rightarrow C$ be $m$ continuous pseudocontractive mappings; let $S_{1}, S_{2}, \ldots, S_{l}$ : $C \rightarrow C$ be l continuous quasi-nonexpansive mappings; let $A_{1}, A_{2}, \ldots, A_{d}: C \rightarrow H$ be $d \gamma_{j-}$ inverse strongly monotone mappings with constants $\gamma_{j} \in(0,1), j=1,2, \ldots, d$; let $f_{1}, f_{2}, \ldots, f_{t}$ : $C \times C \rightarrow \mathbb{R}$ be $t$ bifunctions satisfying conditions (A1)-(A4). Let $F:=\bigcap_{i=1}^{m} \operatorname{Fix}\left(T_{i}\right) \cap$ $\bigcap_{j=1}^{d} A_{j}^{-1}(0) \cap \bigcap_{k=1}^{l} \operatorname{Fix}\left(S_{k}\right) \cap \bigcap_{h=1}^{t} E P\left(f_{h}\right) \neq \emptyset$. Let $\left\{x_{n}\right\}$ be a sequence defined by (3.1). Then the sequence $\left\{x_{n}\right\}_{n \geq 0}$ converges strongly to the element of $F$ nearest to $x_{0}$.

Proof From Lemma 3.1, we obtain that $F \subset C_{n}, \forall n \geq 0$ and $x_{n}$ is well defined for each $n \geq 0$. From $x_{n}=P_{C_{n}}\left(x_{0}\right)$ and $x_{n+1}=P_{C_{n+1}}\left(x_{0}\right) \in C_{n+1} \subset C_{n}$, we obtain that

$$
\left\langle x_{n+1}-x_{n}, x_{n}-x_{0}\right\rangle \geq 0 \text { and }\left\|x_{n}-x_{0}\right\| \leq\left\|x_{n+1}-x_{0}\right\| .
$$

Besides, by Lemma 2.1,

$$
\left\|x_{n}-p\right\|^{2}=\left\|P_{C_{n}} x_{0}-x_{0}\right\| \leq\left\|x_{0}-p\right\|^{2}-\left\|x_{0}-x_{n}\right\|^{2} \leq\left\|x_{0}-p\right\|^{2} .
$$

Thus, the sequence $\left\{\left\|x_{n}-x_{0}\right\|\right\}_{n \geq 0}$ is a bounded nondecreasing sequence of real numbers. So, $\lim _{n \rightarrow \infty}\left\|x_{n}-x_{0}\right\|$ exists. Similarly, by Lemma 2.1 , we have that for any positive integer $\mu$

$$
\begin{aligned}
\left\|x_{n+\mu}-x_{n}\right\|^{2} & =\left\|x_{n+\mu}-P_{C_{n}} x_{0}\right\|^{2} \\
& \leq\left\|x_{n+\mu}-x_{0}\right\|^{2}-\left\|P_{C_{n}} x_{0}-x_{0}\right\|^{2} \\
& =\left\|x_{n+\mu}-x_{0}\right\|^{2}-\left\|x_{n}-x_{0}\right\|^{2} \quad \text { for all } n \geq 0 .
\end{aligned}
$$

Since $\lim _{n \rightarrow \infty}\left\|x_{n}-x_{0}\right\|$ exists, we have that $\lim _{n \rightarrow \infty}\left\|x_{n+\mu}-x_{n}\right\|=0$ and hence, $\left\{x_{n}\right\}_{n \geq 1}$ is a Cauchy sequence in $C$. Therefore, there exists $x^{*} \in C$ such that $\lim _{n \rightarrow \infty} x_{n}=x^{*}$. Since $x_{n+1} \in C_{n+1}$, we have that

$$
\left\|w_{n}-x_{n+1}\right\| \leq\left\|x_{n}-x_{n+1}\right\|
$$

Thus,

$$
\lim _{n \rightarrow \infty}\left\|x_{n+1}-w_{n}\right\|=0
$$


and hence $\left\|x_{n}-w_{n}\right\| \leq\left\|x_{n}-x_{n+1}\right\|+\left\|x_{n+1}-w_{n}\right\| \rightarrow 0$ as $n \rightarrow \infty$, which implies that $w_{n} \rightarrow x^{*}$ as $n \rightarrow \infty$.

Next, we observe that for $p \in F$ and using Lemma 2.3,

$$
\begin{aligned}
\left\|y_{n}-p\right\|^{2} & =\left\|\alpha_{n} x_{n}+\left(1-\alpha_{n}\right) S_{n+1} z_{n}-p\right\|^{2} \\
& =\left\|\alpha_{n}\left(x_{n}-p\right)+\left(1-\alpha_{n}\right)\left(S_{n+1} z_{n}-p\right)\right\|^{2} \\
& =\alpha_{n}\left\|x_{n}-p\right\|+\left(1-\alpha_{n}\right)\left\|S_{n+1} z_{n}-p\right\|^{2}-\alpha_{n}\left(1-\alpha_{n}\right)\left\|x_{n}-S_{n+1} z_{n}\right\|^{2} \\
& \leq \alpha_{n}\left\|x_{n}-p\right\|^{2}+\left(1-\alpha_{n}\right)\left\|z_{n}-p\right\|^{2}-\alpha_{n}\left(1-\alpha_{n}\right)\left\|x_{n}-S_{n+1} z_{n}\right\|^{2} .
\end{aligned}
$$

But

$$
\begin{aligned}
\left\|z_{n}-p\right\|^{2} & \leq\left\|x_{n}-p\right\|^{2}+\lambda_{n}\left(\lambda_{n}-2 \gamma\right)\left\|A_{n+1} x_{n}-A_{n+1} p\right\|^{2} \\
& =\left\|x_{n}-p\right\|^{2}+\lambda_{n}\left(\lambda_{n}-2 \gamma\right)\left\|A_{n+1} x_{n}\right\|^{2} .
\end{aligned}
$$

So, using (3.7) in (3.6), we obtain that

$$
\begin{aligned}
\left\|y_{n}-p\right\|^{2} \leq & \alpha_{n}\left\|x_{n}-p\right\|^{2}+\left(1-\alpha_{n}\right)\left[\left\|x_{n}-p\right\|^{2}+\lambda_{n}\left(\lambda_{n}-2 \gamma\right)\left\|A_{n+1} x_{n}\right\|^{2}\right] \\
& -\alpha_{n}\left(1-\alpha_{n}\right)\left\|x_{n}-S_{n+1} z_{n}\right\|^{2} \\
= & \left\|x_{n}-p\right\|^{2}+\left(1-\alpha_{n}\right) \lambda_{n}\left(\lambda_{n}-2 \gamma\right)\left\|A_{n+1} x_{n}\right\|^{2} \\
& -\alpha_{n}\left(1-\alpha_{n}\right)\left\|x_{n}-S_{n+1} z_{n}\right\|^{2} .
\end{aligned}
$$

Moreover, we obtain that

$$
\begin{aligned}
\left\|w_{n}-p\right\|^{2} & =\left\|\eta \sum_{i=1}^{m} \beta_{i} F_{i, r_{n}} y_{n}+(1-\eta) \sum_{h=1}^{m} \xi_{h} G_{h, r_{n}} y_{n}-p\right\|^{2} \\
& \leq\left\|y_{n}-p\right\|^{2} .
\end{aligned}
$$

Using (3.8) in (3.9) we get that

$$
\begin{gathered}
\left\|w_{n}-p\right\|^{2} \leq\left\|x_{n}-p\right\|^{2}+\left(1-\alpha_{n}\right) \lambda_{n}\left(\lambda_{n}-2 \gamma\right)\left\|A_{n+1} x_{n}\right\|^{2} \\
-\alpha_{n}\left(1-\alpha_{n}\right)\left\|x_{n}-S_{n+1} z_{n}\right\|^{2} .
\end{gathered}
$$

Now, using the fact that $\lambda_{n}<2 \gamma$, inequality (3.10) gives (for some constant $M_{0}>0$ ) that

$$
\alpha_{n}\left(1-\alpha_{n}\right)\left\|x_{n}-S_{n+1} z_{n}\right\| \leq\left\|x_{n}-p\right\|^{2}-\left\|w_{n}-p\right\|^{2} \leq M_{0}\left\|x_{n}-w_{n}\right\| .
$$

Hence, we obtain from inequality (3.11) that

$$
\left\|x_{n}-S_{n+1} z_{n}\right\| \rightarrow 0 \quad \text { as } n \rightarrow \infty .
$$

Moreover, from (3.10) we obtain that

$$
\left(1-\alpha_{n}\right) \lambda_{n}\left(2 \gamma-\lambda_{n}\right)\left\|A_{n+1} x_{n}\right\|^{2} \leq\left\|x_{n}-p\right\|^{2}-\left\|w_{n}-p\right\|^{2} \leq M_{0}\left\|x_{n}-w_{n}\right\|,
$$


which yields that

$$
\lim _{n \rightarrow \infty}\left\|A_{n+1} x_{n}\right\|=0
$$

Now,

$$
\begin{aligned}
\left\|x_{n}-z_{n}\right\| & =\left\|x_{n}-P_{C}\left(x_{n}-\lambda_{n} A_{n+1} x_{n}\right)\right\|=\left\|P_{C} x_{n}-P_{C}\left(x_{n}-\lambda_{n} A_{n+1} x_{n}\right)\right\| \\
& \leq\left\|x_{n}-x_{n}+\lambda_{n} A_{n+1} x_{n}\right\|=\lambda_{n}\left\|A_{n+1} x_{n}\right\| \\
& \leq b\left\|A_{n+1} x_{n}\right\| .
\end{aligned}
$$

It follows from (3.13) and (3.14) that

$$
\lim _{n \rightarrow \infty}\left\|x_{n}-z_{n}\right\|=0
$$

and hence $z_{n} \rightarrow x^{*}$ as $n \rightarrow \infty$.

We now show that $x^{*} \in \bigcap_{k=1}^{l} \operatorname{Fix}\left(S_{k}\right)$. Observe that from (3.12) and (3.15) we obtain that

$$
\left\|S_{n+1} z_{n}-z_{n}\right\| \leq\left\|S_{n+1} z_{n}-x_{n}\right\|+\left\|z_{n}-x_{n}\right\| \rightarrow 0 \quad \text { as } n \rightarrow \infty,
$$

so that

$$
\lim _{n \rightarrow \infty} S_{n+1} z_{n}=x^{*}
$$

Let $\left\{n_{\sigma}\right\}_{\sigma \geq 1} \subset \mathbb{N}$ be such that $S_{n_{\sigma}+1}=S_{1}$ for all $\sigma \in \mathbb{N}$, then since $z_{n_{\sigma}} \rightarrow x^{*}$ as $\sigma \rightarrow \infty$, we obtain from (3.17), using the continuity of $S_{1}$, that

$$
x^{*}=\lim _{\sigma \rightarrow \infty} S_{n_{\sigma}+1} z_{n_{\sigma}}=\lim _{\sigma \rightarrow \infty} S_{1} z_{n_{\sigma}}=S_{1} x^{*} .
$$

Similarly, if $\left\{n_{j}\right\}_{j \geq 1} \subset \mathbb{N}$ is such that $S_{n_{j}+1}=S_{2}$ for all $j \in \mathbb{N}$, then we have again that

$$
x^{*}=\lim _{j \rightarrow \infty} S_{n_{j}+1} z_{n_{j}}=\lim _{j \rightarrow \infty} S_{2} z_{n_{j}}=S_{2} x^{*} .
$$

Continuing, we obtain that $S_{k} x^{*}=x^{*}, k=3, \ldots, l$. Hence, $x^{*} \in \bigcap_{k=1}^{l} F\left(S_{k}\right)$.

Next, we show that $x^{*} \in \bigcap_{j=1}^{d} A_{j}^{-1}(0)$. Since $A_{j}$ is $\gamma$-inverse strongly monotone for $j=$ $1,2, \ldots, d$, we have that $A_{j}$ is $\frac{1}{\gamma}$-Lipschitz continuous. Thus,

$$
\left\|A_{n+1} x_{n}-A_{n+1} x^{*}\right\| \leq \frac{1}{\gamma}\left\|x_{n}-x^{*}\right\| \rightarrow 0 \quad \text { as } n \rightarrow \infty .
$$

Hence, from (3.18) and (3.13), we obtain that

$$
\left\|A_{n+1} x^{*}\right\| \leq\left\|A_{n+1} x_{n}-A_{n+1} x^{*}\right\|+\left\|A_{n+1} x_{n}\right\| \rightarrow 0 \quad \text { as } n \rightarrow \infty .
$$

As a result, we get that

$$
\lim _{n \rightarrow \infty} A_{n+1} x^{*}=0 .
$$


Let $\left\{n_{s}\right\}_{s \geq 1} \subset \mathbb{N}$ be such that $A_{n_{s}+1}=A_{1}$ for all $s \in \mathbb{N}$. Then

$$
A_{1} x^{*}=\lim _{s \rightarrow \infty} A_{n_{s}+1} x^{*}=0 .
$$

Similarly, we have that $A_{j} x^{*}=0$ for $j=2, \ldots, d$. Thus, $x^{*} \in \bigcap_{j=1}^{d} A_{i}^{-1}(0)$.

Furthermore, we show that $x^{*} \in \bigcap_{i=1}^{m} \operatorname{Fix}\left(T_{i}\right)=\bigcap_{i=1}^{m} \operatorname{Fix}\left(F_{i, r}\right), \forall r>0$. Using the fact that $x_{n} \rightarrow x^{*}, z_{n} \rightarrow x^{*}$ as $n \rightarrow \infty$, we obtain that

$$
\begin{aligned}
\left\|F_{1, r_{n}} y_{n}-x^{*}\right\| & \leq\left\|y_{n}-x^{*}\right\| \\
& \leq \alpha_{n}\left\|x_{n}-x^{*}\right\|+\left(1-\alpha_{n}\right)\left\|z_{n}-x^{*}\right\| \\
& \leq\left\|x_{n}-x^{*}\right\|+\left\|z_{n}-x^{*}\right\| \rightarrow 0 \quad \text { as } n \rightarrow \infty .
\end{aligned}
$$

Thus, we obtain from (3.19) that

$$
\lim _{n \rightarrow \infty} F_{1, r_{n}} y_{n}=x^{*}=\lim _{n \rightarrow \infty} y_{n}
$$

This implies that $\lim _{n \rightarrow \infty}\left\|F_{1, r_{n}} y_{n}-y_{n}\right\|=0$. But by Lemma 2.7,

$$
\left\|F_{1, r_{n}} y_{n}-F_{1, r_{0}} y_{n}\right\| \leq \frac{\left|r_{n}-r_{0}\right|}{r_{n}}\left(\left\|F_{1, r_{n}} y_{n}\right\|+\left\|y_{n}\right\|\right) \rightarrow 0 \quad \text { as } n \rightarrow \infty .
$$

Thus,

$$
\lim _{n \rightarrow \infty} F_{1, r_{0}} y_{n}=\lim _{n \rightarrow \infty} F_{1, r_{n}} y_{n}=x^{*}
$$

So, the continuity of $F_{1, r_{0}}$ and the fact that $y_{n} \rightarrow x^{*}$ as $n \rightarrow \infty$ give

$$
x^{*}=\lim _{n \rightarrow \infty} F_{1, r_{0}} y_{n}=F_{1, r_{0}} x^{*} .
$$

A similar argument gives

$$
x^{*}=\lim _{n \rightarrow \infty} F_{i, r_{0}} y_{n}=F_{i, r_{0}} x^{*}, \quad i=2,3, \ldots, m .
$$

Hence,

$$
x^{*} \in \bigcap_{i=1}^{m} \operatorname{Fix}\left(F_{i, r_{0}}\right)=\bigcap_{i=1}^{m} \operatorname{Fix}\left(T_{i}\right)
$$

Moreover, we show that $x^{*} \in \bigcap_{h=1}^{t} E P\left(f_{h}\right)=\bigcap_{h=1}^{t} \operatorname{Fix}\left(G_{h, r_{0}}\right)$. Observe that

$$
\begin{aligned}
\left\|G_{1, r_{n}} y_{n}-x^{*}\right\| & \leq\left\|y_{n}-x^{*}\right\| \\
& \leq \alpha_{n}\left\|x_{n}-x^{*}\right\|+\left(1-\alpha_{n}\right)\left\|z_{n}-x^{*}\right\| \\
& \leq\left\|x_{n}-x^{*}\right\|+\left\|z_{n}-x^{*}\right\| \rightarrow 0 \text { as } n \rightarrow \infty .
\end{aligned}
$$


Thus, we obtain from (3.20) that

$$
\lim _{n \rightarrow \infty} G_{1, r_{n}} y_{n}=x^{*}=\lim _{n \rightarrow \infty} y_{n}
$$

This implies that $\lim _{n \rightarrow \infty}\left\|G_{1, r_{n}} y_{n}-y_{n}\right\|=0$. But by Lemma 2.8 ,

$$
\left\|G_{1, r_{n}} y_{n}-G_{1, r_{0}} y_{n}\right\| \leq \frac{\left|r_{n}-r_{0}\right|}{r_{n}}\left(\left\|G_{1, r_{n}} y_{n}\right\|+\left\|y_{n}\right\|\right) \rightarrow 0 \quad \text { as } n \rightarrow \infty .
$$

Thus,

$$
\lim _{n \rightarrow \infty} G_{1, r_{0}} y_{n}=\lim _{n \rightarrow \infty} G_{1, r_{n}} y_{n}=x^{*} .
$$

So, the continuity of $G_{1, r_{0}}$ and the fact that $y_{n} \rightarrow x^{*}$ as $n \rightarrow \infty$ give

$$
x^{*}=\lim _{n \rightarrow \infty} G_{1, r_{0}} y_{n}=G_{1, r_{0}} x^{*} .
$$

A similar argument gives

$$
x^{*}=\lim _{n \rightarrow \infty} G_{h, r_{0}} y_{n}=G_{i, r_{0}} x^{*}, \quad h=2,3, \ldots, t .
$$

Hence,

$$
x^{*} \in \bigcap_{h=1}^{t} \operatorname{Fix}\left(G_{h, r_{0}}\right)=\bigcap_{h=1}^{t} E P\left(f_{h}\right) .
$$

Finally, we prove that $x^{*}=P_{F}\left(x_{0}\right)$. From $x_{n}=P_{C_{n}}\left(x_{0}\right) n \geq 0$, we obtain that

$$
\left\langle x_{0}-x_{n}, x_{n}-z\right\rangle \geq 0, \quad \forall z \in C_{n} .
$$

Since $F \subset C_{n}$, we also have that

$$
\left\langle x_{0}-x_{n}, x_{n}-p\right\rangle \geq 0, \quad \forall p \in F
$$

So,

$$
\begin{aligned}
0 \leq & \left\langle x_{0}-x_{n}, x_{n}-p\right\rangle=\left\langle x_{0}-x^{*}+x^{*}-x_{n}, x_{n}-x^{*}+x^{*}-p\right\rangle \\
= & \left\langle x_{0}-x^{*}, x_{n}-x^{*}\right\rangle+\left\langle x_{0}-x^{*}, x^{*}-p\right\rangle \\
& +\left\langle x^{*}-x_{n}, x_{n}-x^{*}\right\rangle+\left\langle x^{*}-x_{n}, x^{*}-p\right\rangle \\
\leq & \left\langle x_{0}-x^{*} y, x^{*}-p\right\rangle+\left\|x_{0}-x^{*}\right\|\left\|x_{n}-x^{*}\right\| \\
& +\left\|x_{n}-x^{*}\right\|\left\|x^{*}-p\right\|-\left\|x_{n}-x^{*}\right\|^{2} .
\end{aligned}
$$

Inequality (3.22) implies that

$$
0 \leq\left\langle x_{0}-x^{*}, x^{*}-p\right\rangle+\left(\left\|x_{0}-x^{*}\right\|+\left\|x^{*}-p\right\|\right)\left\|x_{n}-x^{*}\right\| .
$$


By taking limit as $n \rightarrow \infty$ in (3.23), we obtain that

$$
\left\langle x_{0}-x^{*}, x^{*}-p\right\rangle \geq 0, \quad \forall p \in F .
$$

Now, by Lemma 2.2 we have that $x^{*}=P_{F}\left(x_{0}\right)$. This completes the proof.

Remark 3.3 We note that $x^{*}=P_{F}\left(x_{0}\right)$ makes sense since it could be easily shown that $F$ is closed and convex. In fact, it is enough to show that the set of zeros of $\gamma$-inverse monotone mappings and a fixed point set of continuous quasi-nonexpansive mappings are convex sets. Closure of the two sets simply follows from the continuity of the mappings involved.

Remark 3.4 Several authors (see, e.g., $[8,31]$ and references therein) have studied the following problem: Let $C$ be a closed convex nonempty subset of a real Hilbert space $H$ with inner product $\langle\cdot, \cdot\rangle$ and norm $\|\cdot\|$. Let $f: C \times C \rightarrow \mathbb{R}$ be a bifunction and $\Phi: C \rightarrow \mathbb{R} \cup$ $\{+\infty\}$ be a proper extended real-valued function, where $\mathbb{R}$ denotes the set of real numbers. Let $\Theta: C \rightarrow H$ be a nonlinear monotone mapping. The generalized mixed equilibrium problem (abbreviated GMEP) for $f$, $\Phi$ and $\Theta$ is to find $u^{*} \in C$ such that

$$
f\left(u^{*}, y\right)+\Phi(y)-\Phi\left(u^{*}\right)+\left\langle\Theta u^{*}, y-u^{*}\right\rangle \geq 0, \quad \forall y \in C .
$$

The set of solutions for GMEP (3.24) is denoted by

$$
\operatorname{GMEP}(f, \Phi, \Theta)=\{u \in C: f(u, y)+\Phi(y)-\Phi(u)+\langle\Theta u, y-u\rangle \geq 0, \forall y \in C\} .
$$

These authors always claim that if $\Phi \equiv 0 \equiv \Theta$ in (3.24), then (3.24) reduces to the classical equilibrium problem (abbreviated $E P$ ), that is, the problem of finding $u^{*} \in C$ such that

$$
f\left(u^{*}, y\right) \geq 0, \quad \forall y \in C
$$

and $\operatorname{GMEP}(f, 0,0)$ is denoted by $E P(f)$, where

$$
E P(f)=\{u \in C: f(u, y) \geq 0, \forall y \in C\} .
$$

If $f \equiv 0 \equiv \Phi$ in (3.24), then GMEP (1.6) reduces to the classical variational inequality problem and $\operatorname{GMEP}(0,0, \Theta)$ is denoted by $V I(\Theta, C)$, where

$$
V I(\Theta, C)=\{u \in C:\langle\Theta u, y-u\rangle \geq 0, \forall y \in C\} .
$$

If $f \equiv 0 \equiv \Theta$, then GMEP (3.24) reduces to the following minimization problem:

$$
\text { find } u^{*} \in C \text { such that } \Phi(y) \geq \Phi\left(u^{*}\right), \quad \forall y \in C \text {; }
$$

and $\operatorname{GMEP}(0, \Phi, 0)$ is denoted by $\operatorname{Argmin}(\Phi)$, where

$$
\operatorname{Argmin}(\Phi)=\{u \in C: \Phi(u) \leq \Phi(y), \forall y \in C\} .
$$


If $\Theta \equiv 0$, then (3.24) becomes the mixed equilibrium problem (abbreviated $M E P$ ) and $\operatorname{GMEP}(f, \Phi, 0)$ is denoted by $\operatorname{MEP}(f, \Phi)$, where

$$
\operatorname{MEP}(f, \Phi)=\{u \in C: f(u, y)+\Phi(y)-\Phi(u) \geq 0, \forall y \in C\} .
$$

If $\Phi \equiv 0$, then (1.6) reduces to the generalized equilibrium problem (abbreviated GEP) and $\operatorname{GMEP}(f, 0, \Theta)$ is denoted by $\operatorname{GEP}(f, \Theta)$, where

$$
\operatorname{GEP}(f, \Theta)=\{u \in C: f(u, y)+\langle\Theta u, y-u\rangle \geq 0, \forall y \in C\} .
$$

If $f \equiv 0$, then $G M E P$ (3.24) reduces to the generalized variational inequality problem (abbreviated $G V I P)$ and $\operatorname{GMEP}(0, \Phi, \Theta)$ is denoted by $\operatorname{GVIP}(\Phi, \Theta, C)$, where

$$
\operatorname{GVIP}(\Phi, \Theta, C)=\{u \in K: \Phi(y)-\Phi(u)+\langle\Theta u, y-u\rangle \geq 0, \forall y \in C\}
$$

It is worthy to note that if we define $\Gamma: C \times C \rightarrow \mathbb{R}$ by

$$
\Gamma(x, y)=f(x, y)+\Phi(y)-\Phi(x)+\langle\Theta x, y-x\rangle
$$

then it could be easily checked that $\Gamma$ is a bifunction and satisfies properties (A1)-(A4). Thus, the so-called generalized mixed equilibrium problem reduces to the classical equilibrium problem for the bifunction $\Gamma$. Thus, consideration of the so-called generalized mixed equilibrium problem in place of the classical equilibrium problem studied in this paper leads to no further generalization.

\section{Application (convex differentiable optimization)}

In Section 1, we defined a Lipschitz continuous mapping and an inverse strongly monotone mapping. Inverse strongly monotone mappings arise in various areas of optimization and nonlinear analysis (see, for example, [32-38]). It follows from the Cauchy-Schwarz inequality that if a mapping $A: D(A) \subseteq H \rightarrow R(A) \subseteq H$ is $\frac{1}{L}$-inverse strongly monotone, then $A$ is $L$-Lipschitz continuous. The converse of this statement, however, fails to be true. To see this, take for instance $A=-I$, where $I$ is the identity mapping on $H$, then $A$ is $L$ Lipschitz continuous (with $L=1$ ) but not $\frac{1}{L}$-inverse strongly monotone (that is, not firmly nonexpansive in this case).

Baillon and Haddad [39] showed in 1977 that if $D(A)=H$ and $A$ is the gradient of a convex functional on $H$, then $A$ is $\frac{1}{L}$-inverse strongly monotone if and only if $A$ is $L$-Lipschitz continuous. This remarkable result, which has important applications in optimization theory (see, for example, [40-42]), has become known as the Baillon-Haddad theorem. In fact, we have the following theorem.

Theorem 4.1 (Baillon-Haddad) (see Corollary 10 of [39]) Let $\phi: H \rightarrow \mathbb{R}$ be a convex Fréchet-differentiable functional on $H$ such that $\nabla \phi$ is L-Lipschitz continuous for some $L \in(0,+\infty)$, then $\nabla \phi$ is a $\frac{1}{L}$-inverse strongly monotone mapping (where $\nabla \phi$ denotes the gradient of the functional $\phi$ ).

Now, let us turn to the problem of minimizing a continuously Fréchet-differentiable convex functional with minimum norm in Hilbert spaces. 
Let $K$ be a closed convex subset of a real Hilbert space $H$, consider the minimization problem given by

$$
\min _{x \in K} \phi(x)
$$

where $\phi$ is a Fréchet-differentiable convex functional. Let $\Omega \subseteq K$, the solution set of (4.1), be nonempty. It is known that a point $z \in \Omega$ if and only if the following optimality condition holds:

$$
z \in K, \quad\langle\nabla \phi(z), x-z| \geq 0, \quad x \in K .
$$

It is easy to see that if $K=H$, then optimality condition (4.2) is equivalent to $z \in \Omega$ if and only if $z \in(\nabla \phi)^{-1}(0)$.

Thus, we obtain the following as a corollary of Theorem 3.2.

Theorem 4.2 Let $C$ be a nonempty closed convex subset of a real Hilbert space $H$. Let $T_{1}, T_{2}, \ldots, T_{m}: C \rightarrow C$ be m continuous pseudocontractive mappings; let $S_{1}, S_{2}, \ldots, S_{l}: C \rightarrow$ C bel continuous quasi-nonexpansive mappings; let $\phi_{1}, \phi_{2}, \ldots, \phi_{d}: H \rightarrow H$ bed convex and Fréchet-differentiable functionals on $H$ such that $(\nabla \phi)_{j}$ is $L_{j}$-Lipschitz continuous for some $L_{j} \in(0,+\infty), j=1,2, \ldots, d ;$ let $f_{1}, f_{2}, \ldots, f_{t}: C \times C \rightarrow \mathbb{R}$ be $t$ bifunctions satisfying conditions (A1)-(A4). Let $F:=\bigcap_{i=1}^{m} \operatorname{Fix}\left(T_{i}\right) \cap \bigcap_{j=1}^{d}\left(\nabla \phi_{j}\right)^{-1}(0) \cap \bigcap_{k=1}^{l} \operatorname{Fix}\left(S_{k}\right) \cap \bigcap_{h=1}^{t} E P\left(f_{h}\right) \neq \emptyset$. Let $\left\{x_{n}\right\}_{n \geq 0}$ be a sequence defined by

$$
\left\{\begin{array}{l}
x_{0} \in C_{0}=C \quad \text { chosen arbitrarily, } \\
z_{n}=P_{C}\left(x_{n}-\lambda_{n}(\nabla \phi)_{n+1} x_{n}\right), \\
y_{n}=\alpha_{n} x_{n}+\left(1-\alpha_{n}\right) S_{n+1} z_{n}, \\
w_{n}=\eta \sum_{i=1}^{m} \beta_{i} F_{i, r_{n}} y_{n}+(1-\eta) \sum_{h=1}^{t} \xi_{h} G_{h, r_{n}} y_{n}, \\
C_{n+1}=\left\{z \in C_{n}:\left\|w_{n}-z\right\| \leq\left\|x_{n}-z\right\|\right\}, \\
x_{n+1}=\prod_{C_{n+1}}\left(x_{0}\right), \quad n \geq 0,
\end{array}\right.
$$

where $(\nabla \phi)_{n}=(\nabla \phi)_{n(\bmod d)}, S_{n}=S_{n(\bmod l)} ;\left\{r_{n}\right\} \subset(0, \infty)$ such that $\lim _{n \rightarrow \infty} r_{n}=r_{0}>0 ;\left\{\alpha_{n}\right\}_{n \geq 1}$ a sequence in $(0,1)$ such that $\liminf _{n \rightarrow \infty} \alpha_{n}\left(1-\alpha_{n}\right)>0 ;\left\{\beta_{i}\right\}_{i=1}^{m},\left\{\xi_{h}\right\}_{h=1}^{t} \subset(0,1)$ such that $\sum_{i=1}^{m} \beta_{i}=1=\sum_{h=1}^{t} \xi_{h} ; \eta \in(0,1)$ and $\left\{\lambda_{n}\right\}$ is a sequence in $[a, b]$ for some $a, b \in \mathbb{R}$ such that $0<a<b<\frac{2}{L}, L=\max _{1 \leq j \leq d}\left\{L_{j}\right\}$. Then the sequence $\left\{x_{n}\right\}_{n \geq 0}$ converges strongly to the element of $F$ nearest to $x_{0}$.

Proof Since, by our hypothesis, $(\nabla \phi)_{j}$ is $L_{j}$-Lipschitz continuous for some $L_{j} \in(0,+\infty)$, $j=1,2, \ldots, d$, we obtain from Theorem 4.1 that $(\nabla \phi)_{j}$ is $\frac{1}{L_{j}}$-inverse strongly monotone, $j=$ $1,2, \ldots, d$; and since $L=\max _{1 \leq j \leq d}\left\{L_{j}\right\}$, it is then easy to see that $(\nabla \phi)_{j}$ is $\frac{1}{L}$-inverse strongly monotone, $j=1,2, \ldots, d$. The rest, therefore, follows as in the proof of Theorem 3.2 with $\gamma=\frac{1}{L}$. This completes the proof.

The authors declare that they have no competing interests. 


\section{Author details}

1 Department of Mathematics, Nnamdi Azikiwe University, P.M.B. 5025, Awka, Anambra State, Nigeria. ${ }^{2}$ Bahir Dar University, P.O. Box 859, Bahir Dar, Ethiopia. ${ }^{3}$ Department of Mathematics, King Abdulaziz University, P.O. Box 80203, Jeddah, 21589, Saudi Arabia.

\section{Acknowledgements}

This article was funded by the Deanship of Scientific Research (DSR), King Abdulaziz University, Jeddah. N Shahzad acknowledges with thanks DSR for financial support.

Received: 27 August 2013 Accepted: 21 November 2013 Published: 09 Jan 2014

\section{References}

1. Kim, GE: Weak and strong convergence for quasi-nonexpansive mappings in Banach spaces. Bull. Korean Math. Soc 49(4), 799-813 (2012)

2. Dotson, WG: On the Mann iterative process. Trans. Am. Math. Soc. 149, 63-73 (1970)

3. Diaz, JB, Metcalf, FT: On the set of subsequential limit points of successive approximations. Trans. Am. Math. Soc. 135, 459-485 (1969)

4. Kato, T: Nonlinear semigroups and evolution equations. J. Math. Soc. Jpn. 19, 508-520 (1967)

5. Chidume, CE: Geometric Properties of Banach Spaces and Nonlinear Iterations. Lecture Notes in Mathematics, vol. 1965 (2009). ISBN:978-1-84882-189-7

6. Ofoedu, EU, Zegeye, H: Further investigation on iteration processes for pseudocontractive mappings with application. Nonlinear Anal. TMA 75, 153-162 (2012)

7. Blum, E, Oettli, W: From optimization and variational inequalities to equilibrium problems. Math. Stud. 63(1-4), 123-145 (1994)

8. Katchang, P, Jitpeera, T, Kumam, P: Strong convergence theorems for solving generalized mixed equilibrium problems and general system of variational inequalities by the hybrid method. Nonlinear Anal. Hybrid Syst. (2010). doi:10.1016/j.nahs.2010.07.001

9. Zegeye, $\mathrm{H}$, Shahzad, $\mathrm{N}$ : Strong convergence theorems for a solution of finite families of equilibrium and variational inequality problems. Optimization, 1-17, iFirst (2012)

10. Zegeye, $\mathrm{H}$, Shahzad, $\mathrm{N}$ : Convergence theorems for a common point of solutions of equilibrium and fixed point of relatively nonexpansive multi-valued mapping problems. Abstr. Appl. Anal. 2012, Article ID 859598 (2012)

11. Censor, Y, Gibali, A, Reich, S, Sabach, S: Common solutions to variational inequalities. Set-Valued Var. Anal. 20, 229-247 (2012)

12. Censor, Y, Gibali, A, Reich, S: A von Neumann alternating method for finding common solutions to variational inequalities. Nonlinear Anal. 75, 4596-4603 (2012)

13. Ishikawa, S: Fixed point by a new iteration method. Proc. Am. Math. Soc. 44, 147-150 (1974)

14. Mann, WR: Mean value methods in iteration. Proc. Am. Math. Soc. 4, 506-510 (1953)

15. Martines-Yanes, C, Xu, HK: Strong convergence of CQ method for fixed point iteration. Nonlinear Anal. 64, $2400-2411$ (2006)

16. Nakajo, K, Takahashi, W: Strong convergence theorems for nonexpansive mappings. J. Math. Anal. Appl. 279, $372-379$ (2003)

17. Reich, S: Weak convergence theorems for nonexpansive mappings in Banach spaces. J. Math. Anal. Appl. 67, 274-276 (1979)

18. Zegeye, $\mathrm{H}$, Shahzad, N: Strong convergence theorems for a common zero of countably infinite family of $\alpha$-inverse strongly accretive mappings. Nonlinear Anal. 71, 531-538 (2009)

19. Zegeye, $\mathrm{H}$ : Strong convergence theorems for maximal monotone mappings in Banach spaces. J. Math. Anal. Appl. 343, 663-671 (2008)

20. Bauschke, $\mathrm{HH}$, Matouskova, E, Reich, S: Projections and proximal point methods: convergence results and counterexamples. Nonlinear Anal. 56, 715-738 (2004)

21. Kim, TH, Xu, HK: Strong convergence of modified Mann iteration for asymptotically nonexpansive mappings and semigroups. Nonlinear Anal. 64, 1140-1152 (2006)

22. Nilsrakoo, W, Saejug, S: Weak and strong convergence theorems for countable Lipschitzian mappings and its applications. Nonlinear Anal. TMA 69(8), 2695-2708 (2008)

23. Ofoedu, EU, Shehu, Y, Ezeora, JN: Solution by iteration of nonlinear variational inequalities involving finite family of asymptotically nonexpansive mappings and monotone mappings. Panam. Math. J. 18(4), 61-75 (2008)

24. Yang, L, Su, Y: Strong convergence theorems for countable Lipschitzian mappings and its applications in equilibrium and optimization problems. Fixed Point Theory Appl. 2009, Article ID 462489 (2009). doi:10.1155/2009/462489

25. Zegeye, $\mathrm{H}$, Shahzad, N: Strong convergence theorems for a finite family of asymptotically nonexpansive mappings and semigroups. Nonlinear Anal. (2007). doi:10.1016/j.na.2007.11.005

26. Zegeye, $\mathrm{H}$, Shahzad, N: A hybrid approximation method for equilibrium, variational inequality and fixed point problems. Nonlinear Anal. Hybrid Syst. 4, 619-630 (2010)

27. Zegeye, $\mathrm{H}$, Shahzad, N: Approximating common solution of variational inequality problems for two monotone mappings in Banach spaces. Optim. Lett. 5(4), 691-704 (2011)

28. Kopecka, E, Reich, S: A note on alternating projections in Hilbert space. J. Fixed Point Theory Appl. 12, 41-47 (2012)

29. Zegeye, $\mathrm{H}$ : An iterative approximation method for a common fixed point of two pseudo-contractive mappings. ISRN Math. Anal. 2011, Article ID 621901 (2011). doi:10.5402/2011/621901

30. Kassay, G, Reich, S, Sabach, S: Iterative methods for solving systems of variational inequalities in reflexive Banach spaces. SIAM J. Optim. 21, 1319-1344 (2011)

31. Ofoedu, EU: A general approximation scheme for solutions of various problems in fixed point theory. Int. J. Anal. 2013. Article ID 762831 (2013). doi:10.1155/2013/762831

32. Byrne, CL: Applied Iterative Methods. AK Peters, Wellesley (2008)

33. Combettes, PL: Solving monotone inclusions via compositions of nonexpansive averaged operators. Optimization 53, 475-504 (2004) 
34. Dunn, JC: Convexity, monotonicity and gradient processes in Hilbert space. J. Math. Anal. Appl. 53, 145-158 (1976)

35. Lions, PL, Mercier, B: Splitting algorithms for the sum two nonlinear operators. SIAM J. Numer. Anal. 16, 964-979 (1979)

36. Liu, F, Nashed, MZ: Regularization of nonlinear ill-posed variational inequalities and convergence rates. Set-Valued Anal. 6, 313-344 (1998)

37. Tseng, P: Applications of a splitting algorithm to decomposition in convex programming and variational inequalities. SIAM J. Control Optim. 29, 119-138 (1991)

38. Zhu, DL, Marcotte, P: Co-coercivity and its role in the convergence of iterative schemes for solving variational inequalities. SIAM J. Optim. 6, 714-726 (1996)

39. Baillon, JB, Haddad, G: Quelques propriétés des opérateurs angle-bornés et $n$-cycliquement monotones. Isr. J. Math. 26, 137-150 (1977)

40. Combettes, PL, Wajs, VR: Signal recovery by proximal forward-backward splitting. Multiscale Model. Simul. 4, 1168-1200 (2005)

41. Yamada, I, Ogura, N: Hybrid steepest descent method for variational inequality problem over the fixed point set of certain quasi-nonexpansive mappings. Numer. Funct. Anal. Optim. 25, 619-655 (2004)

42. Masad, E, Reich, S: A note on the multiple-set split convex feasibility problem in Hilbert space. J. Nonlinear Convex Anal. 8, 367-371 (2007)

10.1186/1687-1812-2014-9

Cite this article as: Ofoedu et al.: An algorithm for finding common solutions of various problems in nonlinear operator theory. Fixed Point Theory and Applications 2014, 2014:9

\section{Submit your manuscript to a SpringerOpen ${ }^{\circ}$ journal and benefit from:}

- Convenient online submission

- Rigorous peer review

- Immediate publication on acceptance

Open access: articles freely available online

High visibility within the field

- Retaining the copyright to your article 